\title{
Chemomechanical versus drilling methods for caries removal: an in vitro study
}

Kemporn KITSAHAWONG(a) Ana Lucia SEMINARIO(b)

Patimaporn PUNGCHANCHAIKUL(a) Anoma RATTANACHAROENTHUM(a) Waranuch PITIPHAT ${ }^{(c)}$

(a) Khon Kaen University - KKU, Faculty of Dentistry, Pediatric Dentistry, Khon Kaen, Thailand.

(b) University of Washington - UW, School of Dentistry, Pediatric Dentistry, Seattle, WA, USA.

(c)Khon Kaen University - KKU, Faculty of Dentistry, Community Dentistry, Khon Kaen, Thailand.

Declaration of Interests: The authors certify that they have no commercial or associative interest that represents a conflict of interest in connection with the manuscript.

Corresponding Author:

Waranuch Pitiphat

E-mail: waranuch@kku.ac.th

DOI: 10.1590/1807-3107BOR-2015.vol29.0127

Submitted: Dec 16, 2014

Accepted for publication: Jun 23, 2015

Last revision: Aug 31, 2015
Abstract: The purpose of this study was to compare the performance of chemomechanical caries removal (CMCR) with that of conventional drilling for efficacy of caries removal, time spent, morphological changes and microhardness of surface dentin, and microleakage of subsequent restorations. Forty-six carious deciduous molars were randomly divided into two groups: one each for caries removal by (1) CMCR and by (2) drilling. The completeness of caries removal was evaluated by visual and tactile criteria and a caries detector device. Twenty teeth in each group were restored with glass ionomer (GI) and subjected to thermocycling before undergoing microleakage and microhardness tests. In each group, three restored teeth were used for polarized light microscopic analysis, and three unrestored teeth for scanning electron microscopy (SEM). There was no significant difference in the completeness of caries removal between groups. However, time spent for caries removal by CMCR was significantly longer than that required for drilling. Restorations in the CMCR group had significantly more microleakage than those in the drilling group. Dentin hardness of the cavity floor after CMCR was also significantly lower. Microscopic analyses showed roughened and irregular dentin surfaces in the CMCR group, unlike the smooth surfaces observed in the drilling group. In conclusion, CMCR was as efficacious as drilling in term of completeness of caries removal, but required longer excavation times and resulted in lower microhardness of residual dentin as well as more microleakage after restorations with GI. Further laboratory and clinical evaluations on the efficiency and performance of CMCR for the durability of subsequent restorations are required.

Keywords: Dental Caries; Papain; Dentin.

\section{Introduction}

Dental caries continues to affect a significant portion of the world population. Various methods have been used for dental caries management. The conventional method for caries removal is drilling with dental burs. ${ }^{1}$ However, drilling may cause adverse biological reactions to dental pulp tissues and non-selectively remove both infected and sound dental tissues. Importantly, drilling can cause anxiety, fear, and pain in patients and frequently requires a local anesthetic injection. ${ }^{1,2}$ Fear and anxiety in children are known barriers to acceptance of dental treatment. Compliance by some children in dental care can be poor, even with good behavior management. To overcome 
these problems, several conservative caries removal methods have been developed, including air-abrasion, ultrasonic, sono-abration, lasers, and chemomechanical caries removal (CMCR). The advantage of CMCR over other systems is that it does not require sophisticated and costly instruments. Other advantages of CMCR include lower degree of trauma to the tissues, less need for local anesthesia, and reduced chance of dental pulp exposure. $^{3}$

Papacarie ${ }^{\circledR}$ has been introduced as a new method for CMCR. This product contains active ingredients, including papain and chloramines, with bactericidal, bacteriostatic, and anti-inflammatory properties. ${ }^{2,4}$ Papain can specifically digest infected or dead tissues because their collagen molecules are partially degraded and they lack a1-anti-trypsin, which normally inhibits protein digestion in healthy tissues. ${ }^{1,2,4,5}$ Thus, Papacarie ${ }^{\circledR}$ could facilitate the specific removal of carious dentin with minimal damage to healthy dentin., 2,6

Evidence concerning the efficacy of Papacarie ${ }^{\circledR}$ is sparse. Most clinical studies have been small, ,8,9,10,11 with short-term evaluation ${ }^{11}$ and without control groups., ${ }^{3,4}$ Only a few studies have investigated the performance of Papacarie ${ }^{\circledR}$ in vitro, ${ }^{5,6}$ and there are no studies comparing microleakage between Papacarie ${ }^{\circledR}$ and drilling. The aims of this in vitro study were to evaluate the performance of Papacarie ${ }^{\circledR}$ in comparison with the drilling method with regard to: (1) efficacy of caries removal; (2) time spent for treatment; (3) morphological changes in dentin after caries removal as examined by scanning electron microscopy (SEM) and polarized light microscopy; (4) microhardness of the residual dentin; and (5) microleakage after restoration with a glass ionomer $(\mathrm{GI})$.

\section{Methodology}

This study was approved by the Khon Kaen University Ethics Committee for Human Research, Thailand (Protocol Number HE572096), as exempt research. Forty-six human primary molar teeth with cavitated dentinal caries lesions and stored in $0.1 \%$ Thymol solution (Ajax Finechem Pty Ltd., Auckland, New Zealand) were included. Teeth with any pathology other than caries, such as developmental anomalies, fracture, filling material, or root canal treatment, were excluded.

\section{Specimen preparation}

The teeth were randomly divided into two groups: CMCR $(n=23)$ and drilling $(n=23)$. In the CMCR group, caries was removed by means of the Papacarie ${ }^{\circledR}$ gel (F\&A Laboratorio Farmaceutico Ltd., São Paulo, Brasil) according to the manufacturer's instructions. Briefly, the gel was applied for 30-60 s to soften the carious dentin. Dental caries was then gently removed by hand instruments. The procedure was repeated until the gel no longer turned cloudy and the lesion surfaces felt hard. The cavity was wiped with a cotton pellet and rinsed with water spray. In the drilling group, cavities were prepared by means of an aerator with carbide bur no. 330 . Dental caries was then removed by means of an air motor with a steel round bur, followed by a spoon excavator.

\section{Efficacy of caries removal}

A trained operator performed all caries removal and restorations. The efficacy of caries removal was evaluated by tactile and visual inspections performed by another trained dentist, who was blinded to the group status. Each cavity was examined twice in a 2-week interval, with 95\% agreement. For tactile criteria, complete caries removal was defined as a smooth passage of a blunt explorer and the absence of a catch or a "tug-back" sensation., ${ }^{910}$ In addition, the Facelight caries detection device (Proface, W\&H Dentalwerk Burmoos GmbH, Salzburg, Austria) was used to perform visual assessment. The device's function is based on the special fluorescent properties of porphyrin, a metabolic by-product of bacteria in infected dentin. When illuminated with the device, porphyrin will emit red fluorescence, while the sound tooth tissue will show green fluorescence. ${ }^{12}$

\section{Caries removal time}

During the caries removal process, the total excavation time was recorded. In the CMRC group, the time was recorded from the gel application until the completion of the caries removal procedure. In 
the drilling group, the time was recorded from the beginning of drilling until the completion of the caries removal procedure.

\section{Restoration procedures}

Of the 23 teeth in each group, 20 teeth were randomly chosen and restored with GI (GC Fuji IX GP EXTRA, GC Corporation, Tokyo, Japan) before undergoing microleakage and microhardness testing. The remaining 3 teeth were prepared for SEM analysis. The restorative procedures were performed by a single operator according to the manufacturer's instructions. GC dentin conditioner ( $10 \%$ polyacrylic acid solution) was used to clean the tooth surfaces before being restored with GI. Subsequently, all specimens were placed in a thermocycling machine (Medical \& Environment Equipment Research Laboratory, King Mongkut's Institute of Technology, Bangkok, Thailand) with temperature settings at 5 and $55^{\circ} \mathrm{C}$ for 500 cycles, with a 30 -second dwell time in each temperature bath.

\section{Microleakage}

After being thermocycled, each tooth was coated with 3 applications of nail varnish to within $1.0 \mathrm{~mm}$ of the margin of the GI. The crown portion was then immersed in a $0.5 \%$ methylene blue dye solution (Brightchem Sdn Bhd, Pulau Pinang, Malaysia) for $24 \mathrm{~h}$, rinsed with deionized water for $1 \mathrm{~min}$, dried with gauze, and embedded in a clear acrylic resin block (Lang Dental Manufacturing Co., Inc., Wheeling, USA). The specimens were sectioned buccolingually through the GI with a precision diamond saw (IsoMet 1000, Buehler Ltd., Lake Bluff, USA). The sections were examined at 100x magnification with a light diffraction microscope (Stereomicroscope, Nikon measure scope 20, Tokyo, Japan) for the evaluation of dye penetration along the GI margins. Microleakage was scored according to the following criteria: $0=$ no leakage; $1=$ dye penetration limited to enamel; 2 = dye penetration into dentin; and 3 = dye penetration into the pulpal wall. Three measurements of the extent of dye penetration and the average were recorded for each specimen. All specimens were examined by one calibrated examiner.

\section{Microhardness test}

Twenty teeth from each group were prepared for microhardness testing. The Vickers hardness of the residual dentin was determined at distances of $100 \mu \mathrm{m}$ and $200 \mu \mathrm{m}$ from the floor of the cavity lesion, by means of a microhardness testing machine (Matsuzawa Model MXT70, Matsuzawa Seiki Co., Ltd., Tokyo, Japan). The hardness testing was loaded to $50 \mathrm{~g}(0.49 \mathrm{~N})$ for $15 \mathrm{~s}$. Each specimen was measured 3 times by one examiner and averaged.

\section{Polarized light microscopy}

Three of the restored specimens from each group were sectioned longitudinally in a mesio-distal direction through the GI with a diamond saw to a $0.5 \mathrm{~mm}$ thickness. Sections were examined under a polarized light microscope (Nikon Polarizing Microscope ECLIPSE LV100 POL, DS-Fil, Tokyo, Japan).

\section{SEM examination}

Three unrestored teeth from each group were mounted on aluminum stubs, placed in a vacuum chamber, and gold-sputter-coated. Surfaces of the remaining dentin were examined under SEM (Hitachi S3000N, Hitachi Science systems, Osaka, Japan)

\section{Statistical analysis}

Fisher's exact test was used to evaluate the between-group differences in the efficacy of caries removal by clinical criteria and a caries detection device. The Mann-Whitney U test was used to compare the time spent and the length of dye staining in a microleakage study. The analyses were performed with SPSS version 17.0 software (SPSS Inc., Chicago, USA). All statistical tests were two-sided, with a significance level of $5 \%$.

\section{Results \\ Efficacy of caries removal}

The results showed that caries removal was complete in all teeth in the CMCR group, while one tooth in the drilling group was considered incomplete. Additional assessment with the Facelight device found that caries removal was complete in 19 of 23 teeth in the CMCR group and 20 of 23 teeth in the drilling group. There was no significant difference 
in the efficacy of caries removal between the two groups (Fisher's exact test; $\mathrm{p}=1.0$ ).

\section{Caries removal time}

The average time for complete caries removal in the CMCR group was $3.6 \pm 5 \mathrm{~min}$, while it was $1.7 \pm 5 \mathrm{~min}$ in the drilling group. The average time required for CMCR to complete caries removal was significantly higher than that required for drilling (mean difference $=1.9 \pm 0.1 ; 95 \%$ confidence interval, $\mathrm{CI}=1.6-2.2 \mathrm{~min} ; \mathrm{p}<0.001)$.

\section{Microleakage}

The mean length of microleakage in the CMCR group was $2.1 \pm 0.9 \mathrm{~mm}$, while it was $1.2 \pm 0.6 \mathrm{~mm}$ in the drilling group. The difference was statistically significant (mean difference $=0.8 \pm 0.2$; $95 \% \mathrm{CI}=0.3-1.3 \mathrm{~mm} ; \mathrm{p}<0.001)$. Table 1 shows that the percentage of teeth with dye penetration into the pulpal wall was significantly higher in the CMCR group as compared with that in the drilling group (Fisher's exact test; $\mathrm{p}=0.006$ ).

\section{Microhardness test}

The mean Vickers hardness values of residual dentin following caries removal are shown in Table 2. The hardness of residual dentin at indentations of both $100 \mu \mathrm{m}$ and $200 \mu \mathrm{m}$ following caries removal with

Table 1. Microleakage score according to comparison group.

\begin{tabular}{lcccc}
\hline \multirow{2}{*}{ Microleakage score } & \multicolumn{2}{c}{ CMCR } & \multicolumn{2}{c}{ Drilling } \\
& $(\mathrm{n}=20)$ & \multicolumn{2}{c}{$(\mathrm{n}=20)$} \\
\cline { 2 - 6 } & $\mathrm{n}$ & $\%$ & $\mathrm{n}$ & $\%$ \\
\hline $0=$ no leakage & 0 & 0 & 0 & 0 \\
$1=$ dye penetration into enamel only & 1 & 5 & 7 & 35 \\
$2=$ dye penetration into dentin & 13 & 65 & 13 & 65 \\
$3=$ dye penetration into pulpal wall & 6 & 30 & 0 & 0 \\
\hline
\end{tabular}

Table 2. Microhardness of residual dentin after caries removal according to comparison group.

\begin{tabular}{lcc}
\hline \multirow{2}{*}{ Caries removal method } & \multicolumn{2}{c}{ Vickers hardness value (mean \pm SD) } \\
\cline { 2 - 3 } & $100 \mu \mathrm{m}$ & $200 \mu \mathrm{m}$ \\
\hline CMCR $(\mathrm{n}=20)$ & $43.7 \pm 5.2$ & $44.6 \pm 4.4$ \\
Drilling $(\mathrm{n}=20)$ & $47.4 \pm 4.8$ & $50.5 \pm 3.7$ \\
\hline
\end{tabular}

CMCR was significantly lower than that following drilling $(100 \mu \mathrm{m}, \mathrm{p}<0.023 ; 200 \mu \mathrm{m}, \mathrm{p}<0.0001)$.

\section{SEM}

The morphology of residual dentin in the CMCR group showed a rough and irregular surface with a smear layer. SEM images of the drilling group showed less irregularity and a thin smear layer (Figure 1). In some areas, a cracked surface was observed.

\section{Polarized light microscopy}

A polarized light microscopy viewing of the dentin surface after caries removal in the CMCR group showed a rough and irregular surface, compared with a smooth surface in the drilling group (Figure 2). After restoration, polarized light microscopy images showed that GI adapted well to the dentin surfaces in both groups, and no interfacial gap was observed. However, the dentin-GI interfaces in the drilling group were smoother when compared with those of the CMCR group (Figure 3).

\section{Discussion}

Based on the minimal intervention concept, only caries-infected dentin should be removed, while caries-affected dentin can be conserved. ${ }^{13}$ We used both visual and tactile criteria to evaluate the efficacy of caries removal. These criteria are used in routine practice and have been used in previous studies evaluating the efficacy of CMCR. ${ }^{9,10}$ Our definition of complete caries removal is equivalent to a complete removal of the "infected dentin". ${ }^{14}$ A Facelight device was used for further confirmation of the results. We found that the efficacy of caries removal was not significantly different between CMCR and drilling. Remaining dental caries could still be observed on the wall of the cavity, possibly because it was easier to detect and excavate carious dentin on the cavity floor than on the cavity walls. Chaussain-Miller et al. ${ }^{15}$ suggested that caries removal using CMCR is not beneficial for small cavities, due to a lack of access and visibility. Although for this study we selected only teeth with frank cavitation that seemed accessible to hand instruments, some areas might remain inaccessible.

In this study, the mean caries removal time was $3.5 \mathrm{~min}$ for CMCR compared with $1.7 \mathrm{~min}$ for drilling. 

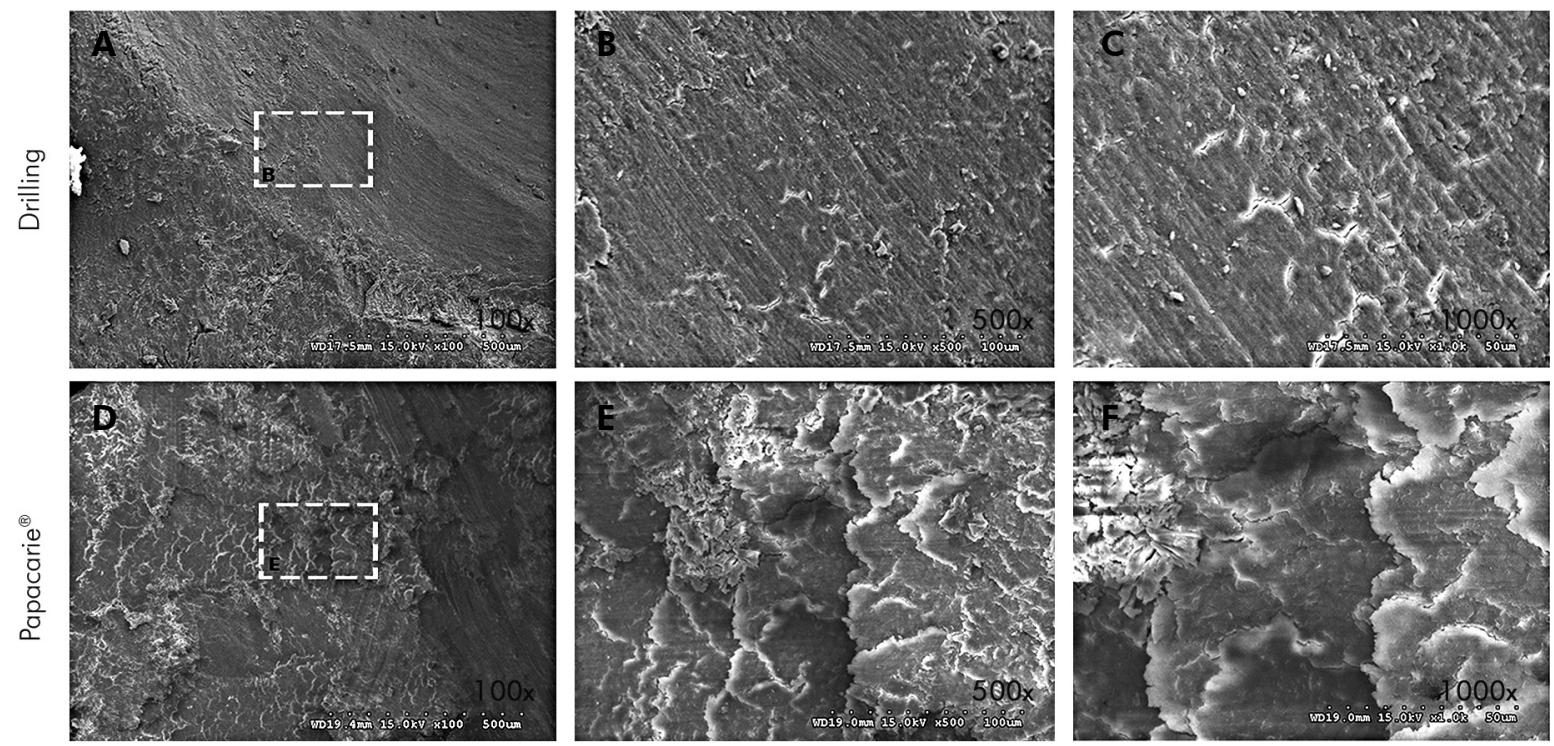

$(A, B, C)$ SEM images of the drilling group at 100x, 500x, and 1000x magnification, respectively. (D,E,F) SEM images of the CMCR group at 100x, 500x, and 1000x magnification, respectively.

Figure 1. SEM images of the residual dentin after caries removal.
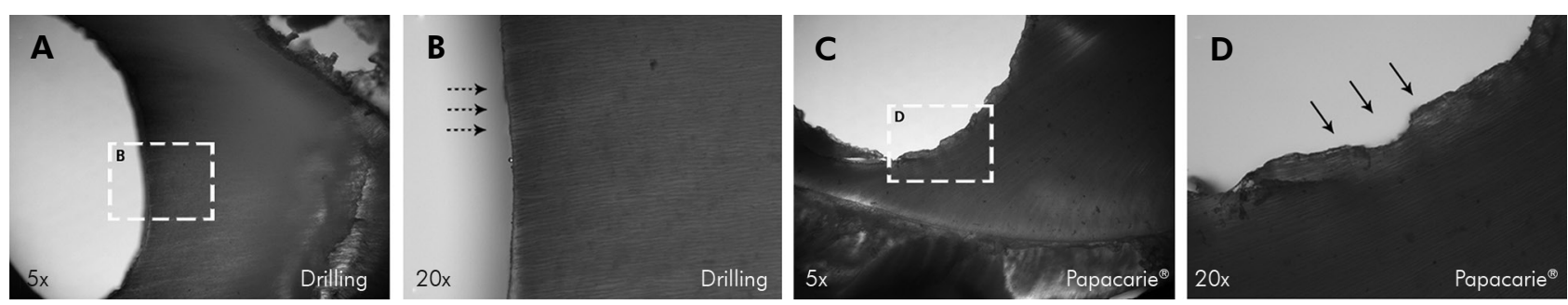

$(A, B)$ Images of the drilling group at $5 x$ and $20 x$ magnification, respectively. (C,D) Images of the CMCR group at 5x and 20x magnification, respectively. Dashed arrow indicates a smooth surface, and solid arrow indicates a rough and irregular surface

Figure 2. Polarized light microscopy images of the residual dentin after caries removal.
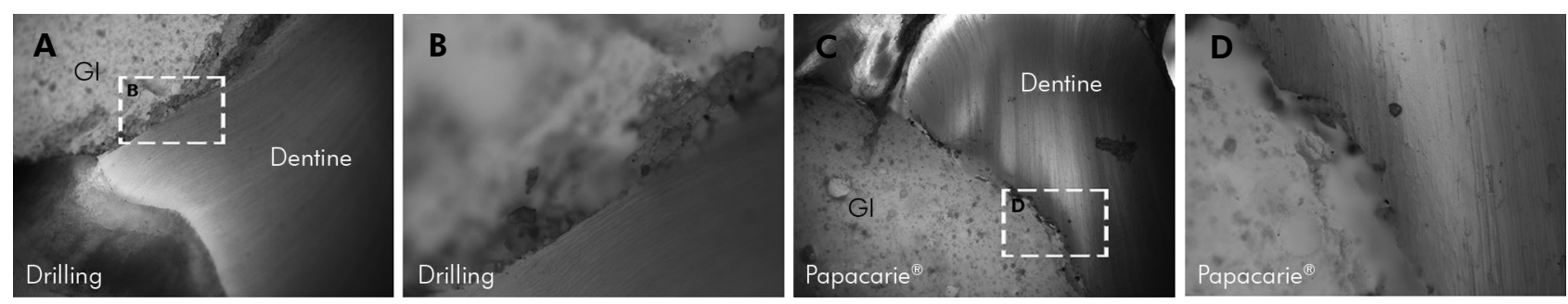

$(A, B)$ Images of the drilling group at $5 x$ and $20 x$ magnification, respectively. (C,D) Images of the CMCR group at 5x and 20x magnification, respectively. GI denotes a glass-ionomer restoration.

Figure 3. Polarized light microscopy images of the interface between residual dentin and $\mathrm{Gl}$.

In previous studies, in both the laboratory ${ }^{6}$ and the clinic, $7,8,9,11$ the duration for excavation by CMCR was significantly longer than that for the drilling method. Our results were within the range of time spent as reported in other in vitro studies, ${ }^{6,16}$ which was generally shorter than that reported in clinical studies. ${ }^{7,9,9,11}$ The longer treatment time can be a disadvantage for CMCR. Nonetheless, the method may 
be suitable for caries management, since anesthesia could be avoided, ${ }^{9,11}$ thus compensating for the time required for anesthesia injection and onset of anesthesia as well as for time needed for behavior management. ${ }^{17,18}$ In addition, caries removal by $\mathrm{CMCR}$ in indirect pulp therapy could decrease the risk of mechanical pulp exposure.

It has been suggested that marginal integrity of the cavity at the tooth-restoration interface contributed greatly to the quality of the cavity seal and the potential for microleakage. ${ }^{19}$ In this study, $95 \%$ of teeth treated with CMCR had microleakage into dentin. Similarly, a previous study reported that treatment of the majority of teeth with Papacarie ${ }^{\circledR}$ resulted in marginal leakage of subsequent restorations. ${ }^{16}$ The mean distance of microleakage in the CMCR group was $2.09 \pm 0.92$ $\mathrm{mm}$. This is in agreement with the results of Khattab and Omar, who reported that GI restoration after Papacarie ${ }^{\circledR}$ treatment showed a high percentage of leakage (50.7 \pm 20.4$)$, with a mean leakage score of $2.7 \pm 0.9 .{ }^{20}$ Polarized light micrographs showed irregular surfaces with an amorphous layer covering the dentinal tubules in the CMCR group. This could be responsible for the marginal leakage of GI restorations, because the irregular surfaces may not be suitable for good adaptation of restorative materials.

The microhardness test of the residual dentin after caries removal showed that the hardness value in the CMCR group was significantly lower than that of the drilling group. This result agrees with that from a previous study. ${ }^{21}$ Furthermore, our results showed that dentin hardness in both groups increased slightly as the distance from the floor of the cavity increased. It has been suggested that residual dentin after caries removal has lower hardness than sound dentine, ${ }^{22}$ and that dentin microhardness increases farther from the infected lesion. ${ }^{23,24}$ Hence, our results further confirm that Papacarie ${ }^{\circledR}$ eliminates only soft infected dentin, while the drilling method removes both infected and affected dentin, and thus likely finishes at sound dentin. Since leaving affected dentin in situ has become more acceptable, ${ }^{19,25}$ Papacarie $^{\circledR}$ has been designed to selectively remove the most damaged layers of the caries lesion and leave the residual dentin for remineralization after the cavity is restored. ${ }^{25}$
The microscopic analyses showed roughened surfaces of dentin after caries removal by CMCR, in contrast to the smooth surfaces remaining after drilling. Moreover, similar to a previous report, our results showed some microcrack lines after CMCR treatment. ${ }^{26}$ This may be due to the hydrophilic nature of the chemomechanical caries removal agent, causing dehydration of the residual dentin. ${ }^{27}$ It is also possible that the crack line is due to the sample dehydration process for examination by SEM. Although previous studies demonstrated that Papacarie ${ }^{\otimes 21,26}$ and its chloramine component are able to remove the smear layer, we observed the presence of smear layers and accumulated debris occluding the dentinal tubules in the CMCR group.

In both groups, the GI adapted well to the dentin surfaces, and no interfacial gap existed. However, the bonding interfaces in the drilling group were smoother compared with those in the CMCR group. Based on the concept of minimal intervention in dentistry, Papacarie ${ }^{\circledR}$ appears to be an alternative caries removal agent that preserves dentinal tissue. However, the excavated cavities have compromised retention form; thus, restorative materials need to rely on chemical bond and require less mechanical retention. Therefore, there is a concern that the loosely attached debris and smear layers with high organic content, as observed in our microscopic analyses of the CMCR group, may also interfere with the bonding ability of adhesive materials. Thus, further research on the bond strength of these restorative materials in cavities prepared with Papacarie ${ }^{\circledR}$ is necessary.

This study had certain limitations. First, the operator could not be blinded, due to the apparent differences of the intervention techniques. Second, the visual and tactile criteria used to evaluate the completion of caries removal are subjective. We therefore used a Facelight device to confirm the results. In addition, the examiners had been trained and calibrated, and the percentage of agreement was high.

This study was conducted in a laboratory setting. A single trained operator performed all the experiments, to minimize inter-operator variability. Thus, variations in the use of these techniques among different operators may affect the outcomes and require future investigation in clinical studies. 


\section{Conclusion}

There was no significant difference in the caries removal efficacy of the CMCR and the drilling methods. However, CMCRrequired a longer excavation time and resulted in rough and irregular dentin surfaces with lower microhardness of residual dentin. Furthermore, there was more marginal microleakage after restoration with GI in the CMCR group. Therefore, CMCR is an effective caries removal method, but further laboratory and clinical evaluations on its efficiency and effects on the durability of subsequent restorations are required.

\section{References}

1. Bittencourt ST, Pereira JR, Rosa AW, Oliveira KS, Ghizoni JS, Oliveira MT. Mineral content removal after Papacarie application in primary teeth: a quantitative analysis. J Clin Pediatr Dent. 2010;34(3):229-31. doi:10.17796/jcpd.34.3.k15t8q1805538524

2. Bussadori SK, Castro LC, Galvão AC. Papain gel: a new chemo-mechanical caries removal agent. J Clin Pediatr Dent. 2006;30(2):115-9. doi:10.17796/jcpd.30.2.xq641w720u101048

3. Bussadori SK, Guedes CC, Bachiega JC, Santis TO, Motta LJ. Clinical and radiographic study of chemical-mechanical removal of caries using Papacarie: 24-month follow-up. J Clin Pediatr Dent. 2011;35(3):251-4. doi:10.17796/jcpd.35.3.75803m02524625h5

4. Bussadori SK, Guedes CC, Hermida Bruno ML, Ram D. Chemo-mechanical removal of caries in an adolescent patient using a papain gel: case report. J Clin Pediatr Dent. 2008;32(3):177-80. doi:10.17796/jcpd.32.3.1168770338617085

5. Piva E, Ogliari FA, Morães RR, Cora F, Henn S, Correr-Sobrinho L. Papain-based gel for biochemical caries removal: influence on microtensile bond strength to dentin. Braz Oral Res. 2008;22(4):364-70. doi:10.1590/S1806-83242008000400014

6. Jawa D, Singh S, Somani R, Jaidka S, Sirkar K, Jaidka R. Comparative evaluation of the efficacy of chemomechanical caries removal agent (Papacarie) and conventional method of caries removal: an in vitro study. J Indian Soc Pedod Prev Dent. 2010;28(2):73-7. doi:10.4103/0970-4388.66739

7. Anegundi RT, Patil SB, Tegginmani V, Shetty SD. A comparative microbiological study to assess caries excavation by conventional rotary method and a chemo-mechanical method. Contemp Clin Dent. 2012;3(4):388-92. doi:10.4103/0976-237X.107420

8. Carrillo CM, Tanaka MH, Cesar MF, Camargo MA, Juliano Y, Novo NF. Use of papain gel in disabled patients. J Dent Child (Chic). 2008;75(3):222-8.

\section{Acknowledgments}

Funding for the study was provided by Khon Kaen University Graduate School (grant 56212102) and the Faculty of Dentistry, Thailand, as well as Fogarty International Center, National Institutes of Health, USA (grant D43 TW007768). Our appreciation is extended to Professor Dr. Timothy A. DeRouen from the University of Washington, Seattle, USA, and Associate Professor Dr. Oranart Matangkasombut from Chulalongkorn University, Thailand, for their valuable suggestions.

9. Kotb RM, Abdella AA, El Kateb MA, Ahmed AM. Clinical evaluation of Papacarie in primary teeth. J Clin Pediatr Dent. 2009;34(2):117-23. doi:10.17796/jcpd.34.2.f312p36g18463716

10. Kumar J, Nayak M, Prasad KL, Gupta N. A comparative study of the clinical efficiency of chemomechanical caries removal using Carisolv and Papacarie - a papain gel. Indian J Dent Res. 2012;23(5):697. doi:10.4103/0970-9290.107429

11. Matsumoto SF, Motta LJ, Alfaya TA, Guedes CC, Fernandes $\mathrm{KP}$, Bussadori SK. Assessment of chemomechanical removal of carious lesions using Papacarie Duo ${ }^{\mathrm{TM}}$ : randomized longitudinal clinical trial. Indian J Dent Res. 2013;24(4):488-92. doi:10.4103/0970-9290.118393

12. Lennon AM, Buchalla W, Brune L, Zimmermann O, Gross U, Attin T. The ability of selected oral microorganisms to emit red fluorescence. Caries Res. 2006;40(1):2-5. doi:10.1159/000088898

13. Frencken JE, Peters MC, Manton DJ, Leal SC, Gordan VV, Eden E. Minimal intervention dentistry (MID) for managing dental caries: a review. Int Dent J. 2012;62(5):223-43. doi:10.1111/idj.12007

14. Banerjee A. Minimal intervention dentistry: part 7. Minimally invasive operative caries management: rationale and techniques. Br Dent J. 2013;214(3):107-11. doi:10.1038/sj.bdj.2013.106

15. Chaussain-Miller C, Decup F, Domejean-Orliaguet S, Gillet D, Guigand M, Kaleka R et al. Clinical evaluation of the Carisolv chemomechanical caries removal technique according to the site/stage concept, a revised caries classification system. Clin Oral Investig. 2003;7(1):32-7. doi:10.1007/s00784-003-0196-5

16. Viral PM, Nagarathan C, Shakuntala BS. Chemomechanical caries removal in primary molars: evaluation of marginal leakage and shear bond strength in bonded restorations: an in vitro study. J Clin Pediatr Dent. 2013;37(3):269-74. doi:10.17796/jcpd.37.3.a56x8502133706nq

17. Maragakis GM, Hahn P, Hellwig E. Clinical evaluation of chemomechanical caries removal in primary molars and its acceptance by patients. Caries Res. 2001;35(3):205-10. doi:10.1159/000047457 
18. Rafique S, Fiske J, Banerjee A. Clinical trial of an air-abrasion/chemomechanical operative procedure for the restorative treatment of dental patients. Caries Res. 2003;37(5):360-4. doi:10.1159/000072168

19. Banerjee A, Kellow S, Mannocci F, Cook RJ, Watson TF. An in vitro evaluation of microtensile bond strengths of two adhesive bonding agents to residual dentine after caries removal using three excavation techniques. J Dent. 2010;38(6):480-9. doi:10.1016/j.jdent.2010.03.002

20. KhattabNM,OmarOM.Papain-based gel for chemo-mechanical caries removal: influence on microleakage and microshear bond strength of esthetic restorative material. J Am Sci. 2012;8(3):391-9.

21. Hamama HH, Yiu CK, Burrow MF, King NM. Chemical, morphological and microhardness changes of dentine after chemomechanical caries removal. Aust Dent J. 2013;58(3):283-92. doi:10.1111/adj.12093

22. Correa FN, Rocha RO, Rodrigues Filho LE, Muench A, Rodrigues CR. Chemical versus conventional caries removal techniques in primary teeth: a microhardness study. J Clin Pediatr Dent. 2007;31(3):187-92. doi:10.17796/jcpd.31.3.1440852707v3g1u0
23. Ogawa K, Yamashita Y, Ichijo T, Fusayama T. The ultrastructure and hardness of the transparent layer of human carious dentin. J Dent Res. 1983;62(1):7-10. doi:10.1177/00220345830620011701

24. Sakoolnamarka R, Burrow MF, Swain M, Tyas MJ. Microhardness and Ca:P ratio of carious and Carisolv treated caries-affected dentine using an ultra-microindentation system and energy dispersive analysis of x-rays: a pilot study. Aust Dent J. 2005;50(4):246-50. doi:10.1111/j.1834-7819.2005.tb00368.x

25. Fusayama T. Two layers of carious dentin; diagnosis and treatment. Oper Dent. 1979;4(2):63-70.

26. Arora R, GoswamiM, ChaudharyS, ChaitraTR, Kishor A, Rallan M. Comparative evaluation of effects of chemo-mechanical and conventional caries removal on dentinal morphology and its bonding characteristics - an SEM study. Eur Arch Paediatr Dent. 2012;13(4):179-84. doi:10.1007/BF03262867

27. Banerjee A, Kidd EA, Watson TF. Scanning electron microscopic observations of human dentine after mechanical caries excavation. J Dent. 2000;28(3):179-86. doi:10.1016/S0300-5712(99)00064-0 